\title{
Philosophiques
}

\section{Réduction, construction, destruction. D'un dialogue à trois : Natorp, Husserl, Heidegger}

\section{Jean-François Courtine}

Volume 36, numéro 2, automne 2009

Edmund Husserl (1859-1938)

URI : https://id.erudit.org/iderudit/039485ar

DOI : https://doi.org/10.7202/039485ar

Aller au sommaire du numéro

\section{Éditeur(s)}

Société de philosophie du Québec

ISSN

0316-2923 (imprimé)

1492-1391 (numérique)

Découvrir la revue

Citer cet article

Courtine, J.-F. (2009). Réduction, construction, destruction. D’un dialogue à trois : Natorp, Husserl, Heidegger. Philosophiques, 36(2), 559-577.

https://doi.org/10.7202/039485ar
Résumé de l'article

In order to introduce the question of tbe " given " and of its elaboration with respect to the motifs of reduction, construction and destruction, we take as a point of departure the first courses of Heidegger at the University of Freiburg in the years 1919-1920. Framed by a sustained debate with the different figures of Neokantianism that occupied the forefront of the philosophical scene in Germany, Heidegger's aim is to take up and to radicalize Husserl's phenomenological enterprise indexed to intuition of an ultimate givenness, through the question : « Was heißt "gegeben”, "Gegebenheit” — dieses Zauberwort der Phänomenologie und der "Stein des Anstoßes" bei den anderen ? " This project of taking Husserl's phenomenlogy will lead Heidegger to reopen the ongoing debate between Husserl and Natorp about the method of reconstruction vs. reduction. So we could venture the hypothesis, according to which the Heideggerian theme of Destruktion "destruction" or

"deconstruction," if it does inherit a sense from the Husserlian Abbau (destruction), from the de-sedimentation of accumulated strata that have come to obstruct the grasp of insight (Einsicht), this theme could all the same be understood as the taking up again - in a practical reversal - Rekonstruktion, Natorpian reconstruction.
Ce document est protégé par la loi sur le droit d'auteur. L'utilisation des services d'Érudit (y compris la reproduction) est assujettie à sa politique d'utilisation que vous pouvez consulter en ligne.

https://apropos.erudit.org/fr/usagers/politique-dutilisation/ 


\title{
Réduction, construction, destruction. D'un dialogue à trois: Natorp, Husserl, Heidegger
}

\author{
JEAN-FRANÇOIS COURTINE \\ Université de Paris-Sorbonne, \\ Archives Husserl de Paris
}

\begin{abstract}
In order to introduce the question of tbe «given» and of its elaboration with respect to the motifs of reduction, construction and destruction, we take as a point of departure the first courses of Heidegger at the University of Freiburg in the years 1919-1920. Framed by a sustained debate with the different figures of Neokantianism that occupied the forefront of the philosophical scene in Germany, Heidegger's aim is to take up and to radicalize Husserl's phenomenological enterprise indexed to intuition of an ultimate givenness, through the question: "Was heißt "gegeben", "Gegebenheit" - dieses Zauberwort der Phänomenologie und der "Stein des Anstoßes" bei den anderen? » This project of taking Husserl's phenomenlogy will lead Heidegger to reopen the ongoing debate between Husserl and Natorp about the method of reconstruction vs. reduction. So we could venture the hypothesis, according to which the Heideggerian theme of Destruktion "destruction" or "deconstruction," if it does inherit a sense from the Husserlian Abbau (destruction), from the desedimentation of accumulated strata that have come to obstruct the grasp of insight (Einsicht), this theme could all the same be understood as the taking up again - in a practical reversal — Rekonstruktion, Natorpian reconstruction.
\end{abstract}

Ces différents motifs: réduction, construction, destruction ont pour commune origine la problématique du donné telle qu'elle se déploie notamment, à la rencontre du néokantisme et de la phénoménologie naissante. Pour le montrer, on peut partir du dossier tel qu'il est établi par Heidegger à l'occasion des deux premiers cours que le jeune assistant de Husserl donne à l'Université de Freiburg dans les années 1919-1920. Il s'agissait d'abord pour lui de proposer comme une première reprise et une radicalisation de l'entreprise husserlienne, indépendamment de tout souci d'orthodoxie ou de ralliement à une école. C'est cette reprise et cette radicalisation qui vont le conduire à rouvrir le débat (en vérité d'actualité) engagé entre Husserl et Natorp ${ }^{1}$.

Le point de départ heideggérien peut être caractérisé par la tentative de radicalisation de la phénoménologie définie comme archi-science de l'origine, comme science pré-théorétique de l'Ur-etwas, comme Vorwissenschaft, puis comme enquête pourtant sur la vie facticielle... C'est en effet dans le cadre de cette détermination de la phénoménologie comme science (l'article de Logos, La philosophie comme science rigoureuse, date de 1911), et dans

1. Cf. l'exposé d'ensemble présenté par Heidegger in GA. 58, Grundprobleme der Phänomenologie, éd. H.-H. Gander, Klostermann, Francfort, 1993, pp. 224 sq: «Das Problem der Gegebenheit - Kritik Natorps und Rickerts ». 
une perspective qu'il est permis de caractériser d'architectonique et de fondationnelle que Heidegger engage à nouveaux frais la discussion autour de la question de l'être-donné (de la Gegebenheit, et du es gibt), thèmes centraux des débats Husserl - Natorp - Rickert — Lask, que Heidegger met ainsi résolument en exergue.

Il n'est évidemment pas question de présenter ici le dossier dans toute sa richesse et complexité - il a déjà fait l'objet d'une riche littérature secondaire - , encore moins de le confronter à d'autres débats ultérieurs relatifs au donné ou au «mythe du donné $»^{2}$. Je me bornerai dans un premier temps à l'examen du débat Husserl - Natorp; puis, dans un second temps, je reviendrai sur l'éclairage heideggérien et son effet de radicalisation.

Au seuil de son cours de 1919 (Die Grundprobleme der Phenomenologie, 1919-1920), Heidegger formule nettement la question directrice et son enjeu:

Qu'est-ce que cela veut dire: «donné», "donation» — ce mot magique de la phénoménologie, qui est aussi une pierre d'achoppement pour les autres orientations philosophiques ?33

En un sens, c'est en tant que phénoménologue attentif au problème méthodologique ou à la première définition de la phénoménologie comme méthode que le jeune Heidegger entend relancer la question de la donation et de l'être-donné, pour autant que cette question est au centre même du projet husserlien de psychologie descriptive indexée sur l'intuition. Mais si Heidegger commence bien par réaffirmer un principe de la fidélité à l'égard de Husserl, voire de l'école phénoménologique en général, sa liberté critique en vue de répéter et de radicaliser le projet phénoménologique n'en est pas moins clairement formulée d'emblée:

C'est de la manière la plus radicale que le radicalisme de la phénoménologie doit s'exercer, aussi bien contre elle-même que contre tout ce qui s'affirme comme connaissance phénoménologique ${ }^{4}$.

Au premier rang des problèmes, puisés à la source, que doit affronter le chercheur qui entend s'engager en phénoménologie figure celui de la délimitation du domaine de l'enquête, de sa scientificité, et surtout — c'est le point qui nous intéressera plus particulièrement ici — de son ultime instance

2. Cf. notamment la discussion ouverte dans le monde anglo-saxon par la série des conférences de Wilfrid Sellars, en 1956, Empiricism and the Philosophy of Mind, Harvard University Press, $1997^{2}$.

3. GA., 58, 5: «Was heißt «gegeben", «Gegebenheit» — dieses Zauberwort der Phänomenologie und der «Stein des Anstoßes» bei den anderen.»

4. GA, 58, 6: «Am radikalisten hat sich aber der Radikalismus der Phänomenologie auszuwirken gegen sie selbst und alles, was als phänomenologische Erkenntnis sich äußert. » 
de légitimation, de son assignation au donné ou au présent-donné dans une évidence irrécusable. Or mettre à l'épreuve ce mot-magique: Gegebenheit, qui a pu servir de slogan, c'est aussi ouvrir le débat avec d'autres écoles, au premier rang desquelles les néokantismes, celui de Marbourg, représenté par Herman Cohen et Paul Natorp, principalement, et celui de l'école de Bade, ici représentée par Heinrich Rickert et Emil Lask.

Ainsi la question du donné et de la donation trouve tout naturellement sa place dans un cours consacré à la détermination de la philosophie (Zur Bestimmung der Philosophie), distribué en deux volets: 1. Die Idee der Philosophie and das Weltanschauungsproblem, 2. Phänomenologie und transzendentale Wertphilosophie, et consacré, qui plus est, à sa détermination comme Urwissenschaft, archi-science de l'originaire. La question directrice qui sera relancée dans le cours de 1919-1920 (Grundprobleme der Phänomenologie) est en effet massivement la suivante: Qu'est-ce que la phénoménologie? La réponse vient aussitôt: À son Idée se trouve indexée cette exigence: science originaire du vivre en et pour soi $i^{5}$. Il faudrait s'arrêter sur le déplacement décisif de l'Erlebnis au Leben an und für sich. Quoi qu'il en soit de ce dernier point qu'il me faut laisser de côté, c'est en fonction de cette «idée » que se pose d'emblée la question du "donné», directement articulée à cette autre interrogation: quel est le domaine d'investigation de la phénoménologie (Forschungsgebiet) ? Ce domaine est-il lui-même "donné » ou «pré-donné » (gegeben, vorgegeben) ? Donné directement ou purement et simplement, sans intermédiaire, sans médiation? Ou, à l'inverse, est-ce que ce domaine originaire (très précisément celui de l'origine: Ursprungsgebiet) ne peut jamais être donné, mais toujours seulement et d'abord à «conquérir» (ibid., 29).

Le manuscrit du premier cours de Fribourg est aujourd'hui incomplet, mais nous pouvons lire dans une Nachschrift due à un auditeur, O. Becker (ibid., 203), cette formulation tout à fait frappante:

Le domaine originaire de la philosophie ne saurait être une proposition ultime, un axiome. [...] Ce domaine originaire ne nous est pas donné. [...] Il n'est jamais donné dans la vie en soi. Il doit toujours être saisi à neuf, à nouveaux frais ${ }^{6}$.

On pourrait sans doute voir, dans cette problématique de l'accès et dans cette exigence d'une saisie à chaque fois renouvelée d'un donné jamais donné entièrement ni surtout définitivement, un des principaux motifs de ce que Heidegger thématisera expressément dans les cours qui suivent

5. «Was ist Phänomenologie? Als ihre Idee ist angesetzt: absolute Ursprungswissenschaft von Leben an und für sich» $(G a .58,171)$.

6. «Das Ursprungsgebiet der Philosophie ist kein letzter Satz, kein Axiom. [...] Das Ursprungsgebiet ist uns nicht gegeben. [...] Das Ursprungsgebiet ist wesentlich nie gegeben im Leben an sich. Er muß immer von Neuem erfaßt werden. » 
immédiatement au titre de la répétition et de la destruction (Wiederholung, Destruktion). J'y reviendrai très rapidement pour conclure cette étude.

Cette question de la donation ultime et des conditions d'accès au donné est d'autant plus importante et décisive, comme nous l'avons déjà indiqué, que l'on prétend prendre au sérieux l'idée de la phénoménologie comme Urwissenschaft. Qu'en est-il ici de l'archi- ou de l'arkhè, de l'Ur- de cette science originelle - ou science de l'origine? Est-il possible, et dans ce cas comment, d'établir méthodiquement le chemin susceptible de reconduire du non-originel à l'originel, à l'Ursprung? Le jeune Heidegger, s'il récuse d'emblée le caractère théorétique, voire pré-théorétique de cette science originaire, Ursprungswissenschaft, c'est-à-dire toujours normé par le théorétique $^{7}$, n'en maintient pas moins l'idée d'une véritable science originaire (eine echte Ur-wissenschaft), de laquelle le théorétique lui-même tirerait son origine. Cette science de l'origine doit être appréhendée de manière à ce que non seulement elle n'ait pas besoin de faire des présuppositions, comme l'exigeait déjà le principe husserlien de la Voraussetzungslosigkeit, rappelé dans l'introduction des Recherches logiques, mais encore à ce qu'elle ne puisse même pas le faire dans la mesure où elle ne sera pas une théorie.

Le coup de maître - ou si l'on préfère le coup de force de Heidegger ici - , celui qui va commander toute la dramatisation de la problématique du donné ultime, passe par un autre déplacement significatif de la question qui ne porte plus tant sur "ce qu'il y a " (on what there is), que sur l'élucidation du "Frageerlebnis » lui-même, du vécu-de-la-question propre à l'interrogation ultime: gibt es etwas? Y a-t-il quelque chose? ou mieux "quelque chose est-il donné ?» Question apparemment minimaliste ou élémentaire dont Heidegger souligne lui-même l'insignifiance et la "pauvreté » (Kümmerlichkeit) —, mais qui est également une question cruciale, celle qui décide de la vie ou de la mort de la philosophie! question qui permet enfin et pour la première fois d'accomplir un saut... «dans le monde». Je cite ce passage tout à fait frappant:

Nous nous tenons ici à la croisée des chemins, devant un choix qui décide de la vie ou de la mort de la philosophie en général, <nous nous tenons > au bord de l'abîme: ou bien nous nous engageons dans le néant, c'est-à-dire dans l'absolue positivité chosique (Sachlichkeit), ou bien nous parvenons à sauter dans un autre monde, ou plus exactement: à sauter enfin dans le monde absolument parlant $^{8}$.

7. Cf. GA. 56/57, 59: «Diese Vorherrschaft des Theoretischen muß gebrochen werden...

8. GA. 56/57, 63: «Wir stehen an der methodischen Wegkreuzung, die über Leben oder Tod der Philosophie überhaupt entscheidet, an einem Abgrund: entweder ins Nichts, d.h. der absoluten Sachlichkeit, oder es gelingt der Sprung in eine andere Welt, oder genauer: überhaupt erst in die Welt » 
On retrouve dans le cours de l'année suivante (Grundprobleme der Phänomenologie, 1919-1920) la même problématique de «la donation ultime comme question» également accentuée dans sa radicalitée. J'ai déjà cité le début de ce passage dont je poursuis la lecture:

Le problème de la donation (être-donné, Gegebenheit) n'est pas un problème particulier relevant d'une recherche spécialisée (spezialistisches Sonderproblem). Avec lui les chemins de la théorie moderne de la connaissance se séparent entre eux <entendez: ceux de l'école de Marbourg d'un côté, ceux de l'école de Bade de l'autre>, et en même temps ils se séparent ensemble de la phénoménologie à qui il appartient de délivrer préalablement ce problème d'une problématique rétrécie, celle de la théorie de la connaissance ${ }^{10}$.

Pour avancer dans l'élaboration de cette problématique de la Gegebenheit, Heidegger, dans une démarche critique à l'égard de Natorp d'un côté et de Rickert de l'autre, rappelle d'abord les distinctions husserliennes classiques entre les différents modes de données qui correspondent aussi aux différentes modalités de la visée intentionnelle ${ }^{11}$ : on distinguera ainsi ce qui est auto-donné (selbstgegeben) en chair et en os (leibhaftig), ce qui est donné soi-même (dans son ipséité) mais non pas leibhaftig, et enfin ce qui ni autodonné, ni donné en chair et en os, à savoir ce qui est donné «bloß symbolisch "; puis on distinguera aussi le donné au sens de "ce que je me donne" (c'est-à-dire en réalité - nous le verrons - le von mir Gesetztes) et le donné au sens du «mir (von außen) vorgegeben» (ibid., 224); en d'autres termes, le donné au sens du "je me donne» le donné, comme posé, et le donné comme trouvé du dehors, pré-donné ${ }^{12}$.

À ces distinctions peuvent correspondre aussi, ce que Heidegger caractérise comme les «deux traitements typiques du problème de la donation, dans l'école de Marbourg et chez Rickert»(GA. 58, 131). Pour les marbourgeois, "on ne saurait jamais parler d'un objet achevé et donné. Avant toute donation se dresse le penser et sa légalité propre, pour laquelle seulement un objet peut être donné. Ainsi un objet donné n'est jamais premier, sa position renvoie toujours à une fonction de pensée originaire. » L'objet s'entend dès lors comme "objet $=x$ ", c'est-à-dire comme "tâche (Aufgabe) d'une position progressant à l'infini.»

9. GA., 58, Grundprobleme der Phänomenologie (1919-1920), 131.

10. Cf. Nachschrift GA. 58, 224 «das Problem der Gegebenheit ist kein spezialistisches Sonderproblem. An ihm scheiden sich die Wege der modernen Erkenntnistheorie unter sich und zugleich von der Phänomenologie, die das Problem vor allem au seiner verengenden erkenntnistheoretischen Problematik loslösen muß» (cf. aussi la Nachschrift d'Oskar Becker, ibid., 221).

11. Ich kann im Leben auf etwas gerichtet sein, ohne daß ich das, worauf ich gerichtet bin, im Charakter der Gegebenheit, des Präsentseins mir gegenüber stehend habe.

12. Cf. GA. 58, les notes d'O. Becker, 224: «Es ist zu scheiden: a) «Gegebensein » im Sinne des von mir Gesetzten, d.h. der Fall, wo ich mir etwas «gebe». b) «Gegeben" im Sinn des mir (von außen) Vorgegeben.» 
Dans un autre appendice de ce même cours ${ }^{13}$, Heidegger, en renvoyant au compte rendu que Natorp venait de publier, dans les Kanstudien, du livre de Bruno Bauch consacré à Kant ${ }^{14}$, pouvait résumer de manière drastique la position de l'école: «Être conscient, conscience (Bewußtsein), cela signifie: penser, déterminer, poser un objet. Chaque donné n'est tel qu'autant qu'il est déterminé dans le penser. C'est seulement à partir de cette détermination que surgit pour la première fois la donation (Gegebenheit). La position-de-la-pensée conserve donc un primat absolu. Connaître, c'est déterminer un objet, poser dans le penser. Ainsi, il n'y a rien de prédonné (es gibt nichts Vorgegebenes). Il n'y a (es gibt) d'objets que dans le penser et, parce que le connaître est un processus qui, en principe, ne connaît pas de fin, l'objet n'est jamais donné, mais seulement son Idée.»

Il n'en demeure pas moins - et c'est là encore un point décisif — sur lequel Heidegger n'aura cessé d'insister dès son Habilitationschrift, en référence directe cette fois à Emil Lask, que le penser ainsi caractérisé comme «poser» ou «déterminer» requiert nécessairement un «quelque chose» (etwas) à déterminer, un quelque chose qui constitue comme un ultime "prédonné » irréductible, un dernier "reste» (ein letzter Rest). Il est d'ailleurs assez significatif de noter que Natorp lui-même, dans les réflexions qu'il rédige à la suite de la lecture de la Logik der reinen Erkenntnis de Cohen, réagit négativement à cette quasi-élimination de tout donné ou pré-donné. Pour Cohen en effet, note-t-il, "c'est en elle-même et exclusivement que la pensée pure doit produire des connaissances pures», ou encore: «Il n'y a que la pensée elle-même qui peut produire ce qui doit valoir comme être.» Voilà qui aboutit à une élimination complète de tout donné. Si donc Natorp accorde que "le "donné" ne saurait avoir de sens que comme Aufgabe, comme tâche ", c'est pour ajouter aussitôt cette restriction capitale:

mais selon le sens même de la tâche ainsi établie — tâche que le penser doit d'abord résoudre -, le donné demeure cependant, et certes à titre de préalablement donné (voraus Gegebenes). Dans son aversion tout à fait légitime contre le pseudo - «donné », Cohen en vient à courir le risque de passer également par dessus ce sens authentique de la donation ${ }^{15}$.

Critique interne à l'école de Marbourg donc, que l'on retrouve aussi dans l'ouvrage de 1910: Die logischen Grundlagen der exakten Wissens-

13. Appendice A: "Gegebenheit» in der Marburger Schule — la donation dans l'école de Marbourg.

14. Kantstudien, XXII, pp. 426-459.

15. Cf. le volume Cohen und Natorp, t. 2, p. 21, éd. H. Holzey, Schwabe, Bâle-Stuttgart 1986 (extrait de $Z u$ Cohens Logik): «aber im Sinne der gestellten Aufgabe, die vom Denken erst zu lösen, bleibt es doch das Gegebene, und zwar voraus Gegebene. In der begründeten Abwehr gegen das falsche "Gegebene" kommt Cohen in Gefahr auch diesen echten Sinne der Gegebenheit zu übersehen.» 
chaften $^{16}$, où Natorp demande encore plus précisément : "Que devrait donc être ce préalablement donné à la connaissance ${ }^{317}$ » Et après avoir récusé les réponses standard: Vorstellung, Empfindung voire Mannigfaltiges, représentation, sensation, multiplicité qui elle-même requiert toujours déjà, à son tour, un acte de détermination, il poursuivait:

C'est bien plutôt cet $x$ qu'il faut tout d'abord déterminer grâce à la pensée, cet $x$ en tant que multiple, tout comme doit l'être aussi d'un autre côté l'unité. Pour la pensée, il n'y a (es gibt) aucun être qui ne soit posé au sein de la pensée elle-même. Penser ne signifie rien d'autre que: poser que quelque chose est: Quant à savoir ce qui est en dehors de et antérieurement à cet être, voilà une question qui n'a absolument aucun sens acceptable ${ }^{18}$.

Dans son étude plus ancienne "Fondation objective et subjective de la connaissance», Philosophische Monatshefte, 1887, pp. 164-165 ${ }^{19}$, Natorp rapprochait cet $x$ déterminable ou à déterminer du dynamei on aristotélicien:

Le donné n'est le concret du phénomène qu'en tant qu'il est préalablement à déterminer, en tant que $x$ déterminable, analogue en cela au dynamei on aristotélicien. Il n'est donné qu'au sens d'une tâche à effectuer, et nullement comme un datum de la connaissance, à partir duquel quelque chose d'autre, encore inconnu, se laisserait déterminer.

Il devient de plus en plus clair que le "positif », le prétendu donné initial est, en fait, ce qui est recherché; c'est pourquoi il vaut mieux parler de recherché ultime. [...] Or de cet absolument "dernier» on a fait un premier, on a pris le quaesitum pour un datum, et par là, on a franchement dénaturé la tâche de la connaissance.

$<$ Mais> si toute détermination est avant tout production de la connaissance, alors on ne peut refuser de réfléchir à ceci : antérieurement à cette production, quelque chose a dû être donné, comme un originaire subjectif, comme un immédiat, qui doit être déterminé et qui, par là, doit être amené à l'objectivité.

- De fait, quelque chose est donné antérieurement à la production de la connaissance: il s'agit de la tâche. Aussi pourrait-on tout aussi bien dire: l'objet serait donné; c'est-à-dire donné comme ce qui est initialement à déterminer, comme un $x$, et non comme une grandeur connue.

Ce «donné ultime» serait-il, demande Natorp, un «originaire subjectif », un "phénomène de dernière instance »? dans lequel il serait permis de voir l'«immédiat de la conscience (subjective)». Est-il permis de "poser

16. 1910, p. 48 (reprint Edition Classic Verlag Dr. Müller, Saarbrücke, 2006).

17. "Was sollte das voraus Gegebene der Erkenntnis denn sein?»

18. «Es ist vielmehr dasjenige $X$, welches als Mannigfaltiges, ebenso wie andererseits als Einheit, durch das Denken erst zu bestimmen ist. [...] Es gibt für das Denken kein Sein, das nicht im Denken selbst gesetzt würde. Denken heißt nichts Anders als: setzen, daß etwas sei; und was außerdem und vordem dies Sein — sei, ist eine Frage, die überhaupt keinen angebaren Sinn hat.»

19. Trad. fr. in Néokantismes et théorie de la connaissance, Paris, Vrin 2000, 136-137. 
cet immédiat de la conscience comme datum immédiat et originaire de la connaissance » ? À quoi Natorp objecte: «il convient plutôt de se demander si cet originaire peut être atteint au moyen de la connaissance. La subjectivité comme telle ne se laisse pas saisir dans son immédiateté. " Elle ne saurait être appréhendée qu'après-coup, dans ses prestations (Leistungen), ou dans ses produits, au titre d'une démarche qu'il faudra caractériser comme «reconstruction ». Dès lors, avant tout concept, le niveau de la "pure » subjectivité serait celui de «l'absolue indétermination». Et Natorp de préciser: "On peut certes remonter à une telle subjectivité comme au chaos originaire, mais on ne peut l'appréhender en soi." La production constructive, objectivante, de la connaissance est absolument préalable: c'est toujours à partir d'une production antérieure que nous reconstruisons, pour autant que cela soit possible, le niveau de la subjectivité originaire; indépendamment de cette voie reconstructrice, et de cette première base qu'est la construction objective initialement effectuée, la subjectivité ne saurait être atteinte par aucune connaissance.

Heidegger résumait quant à lui, fidèlement, mais de manière drastique, la thèse marbourgeoise en ces termes:

La pensée théorique et en particulier la pensée mathématique est «le véritable sens de la conscience». Il appartient à la conscience de «penser, déterminer, poser un objet ». Tout donné n'est donné qu'en tant qu'il est déterminé dans le penser. C'est seulement à partir de cette détermination que surgit la donation. La position propre au penser conserve donc un privilège absolu. Le connaître est détermination objectuelle, poser dans le penser. Il n'y a rien de prédonné. Il n'y a d'objets que dans le penser et parce que le connaître est un procès principiellement sans fin, l'objet n'est jamais donné, mais seulement son Idée (seule la fiction d'un processus de connaissance mené à son terme donne l'objet $)^{20}$.

Ou encore, ibid., 132:

On ne peut jamais parler d'un objet achevé et donné. Antérieurement à toutes données se tient la pensée et sa légalité, pour laquelle seule un objet peut être «donné ${ }^{21}$

20. Grundprobleme der Phänomenologie GA. 58, 224: «Denken, Bestimmen, Setzen eines Gegenstandes. Jedes Gegebene ist nur als im Denken bestimmt gegeben. Aus dieser Bestimmung entspringt erst die Gegebenheit. Die Denksetzung hat einen absoluten Vorrang. Das Erkennen ist Gegenstandsbestimmung, Setzen im Denken. Es gibt nichts Vorgegebenes. Es gibt Gegenstände erst im Denken und weil das Erkennen ein prinzipiell endloser Prozeß ist, ist der Gegenstand nie gegeben, sondern nur seine Idee (erst die Fiktion des ans Ende gelangten Erkenntnisprozesses gibt den Gegenstand). <souligné par moi>

21. «Es ist nirgends von einem fertigen und gegebenen Gegenstand zu reden. Vor allen Gegebenheiten steht das Denken und seine Gesetzlichkeit, für welche allein ein Gegenstand «gegeben» sein kann.» 
J'en viens maintenant à mon second point principal: Husserl Natorp, en prenant plus précisément en vue les deux comptes rendus rédigés par Natorp et portant respectivement sur les Prolégomènes à la logique pure, et sur les Ideen I.

Dans les Recherches logiques, Prolégomènes à la logique pure, Husserl se référant à l'article de 1887: "Über objektive und subjektive Begründung der Erkenntnis ", ainsi qu'à l'Einleitung in die Psychologie de 1888, avait rendu hommage à Natorp et salué sa contribution à la délimitation du champ de la logique pure, en citant une remarque un peu plus tardive du même Natorp ${ }^{22}$ selon laquelle "les lois logiques disent aussi peu comment on pense en fait, dans telles ou telles circonstances ", que comment on doit penser; il avait également souligné «l'influence stimulante» exercée sur lui par les deux textes que je viens de citer (L.U., A, 156). Dans l'important article de $1887^{23}$, Natorp avait de son côté critiqué Ernst Mach et son projet ancien d'une "physique phénoménologique ${ }^{24}$ qui prétendait partir d'un ultime datum subjectif, sur lequel il était permis de s'interroger: Que veut dire ici «donné»? Le donné est-il "connu», «su»? — demandait Natorp. Bien loin de représenter un élément ultime, ce prétendu donné doit être préalable-

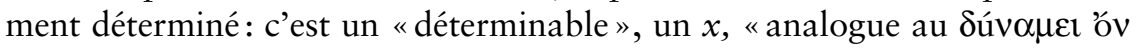
aristotélicien ». Et nous retrouvons ici ce qui va constituer un leitmotiv dans la critique natorpienne de l'immédiateté de la donation: le donné (das Gegebene) n'est donné qu'au sens d'une "tâche" (Aufgabe) à effectuer: ce n'est pas un "datum de la connaissance, à partir duquel quelque chose d'autre, encore inconnu, se laisserait déterminer. " La structure générale de l'argument est ici clairement établie, qui oppose un quelque chose d'indéterminé $=x$, au processus de la connaissance et de la pensée comme Bestimmung, détermination ou mieux production. On doit tout juste reconnaître au «positivisme» dont se réclame Ernst $\mathrm{Mach}^{25}$ un «juste pressentiment»: si toute détermination est une "production» de connaissance, il importe de prendre en considération ce fait que, "antérieurement à toute production, quelque chose devait être donné comme un originaire subjectif < je souligne à nouveau le passage déjà cité > ", une donnée immédiate, mais qui doit précisément être reconduite à l'objectivité. Voilà la «tâche»! L'erreur de principe du positivisme est justement de poser «cet originaire de la connaissance

22. Sozialpsychologie, Stuttgart, 1899, $\mathbb{4} 4$.

23. "Über objektive und subjektive Begründung der Erkenntnis", Philosophische Monatshefte, 1887; trad. fr. I. Thomas-Fogiel, in Cohen, Natorp, Cassirer, Rickert, Windelband, Lask, Cohn, Néokantismes et théorie de la connaissance, Paris, Vrin 2000.

24. Cf. la définition de ce projet dans l'ouvrage de 1872: Die Geschichte und die Wurzel des Satzes von der Erhaltung der Arbeit.

25 Cf. Mandfred Sommer, Denkökonomie und Empfindungstheorie bei Mach und Husserl - Zum Verhältnis von Positivismus und Phänomenologie, in Ernst Mach, Werke und Wirkung, Rudolf Haller et Friedrich Stadler éd., Hölder-Pichler-Tempsky, Vienne, 1988, pp. 309328. 
comme un datum immédiat et premier ", alors que précisément "la subjectivité comme telle ne se laisse pas déterminer dans son immédiateté ». Dans son immédiateté, elle n'est rien d'autre qu' "absolue indétermination absolue » ou, comme le dit encore Natorp, «chaos originel ». Pour accéder à ce niveau de la "subjectivité originaire», il faut précisément un travail médiat de «production» et de «construction» qui est toujours objectivation:

Hormis par cette voie reconstructrice, et sur la base de la construction initialement effectuée, la subjectivité ne pourrait être atteinte par aucune connaissance... Le subjectif n'est premier que pour autant que la tâche de la connaissance est d'abord posée comme résolue; mais ce subjectif ne saurait être un donné, au sens d'un datum pour la connaissance.

Dans son article de 1887, Natorp ne vise évidemment en rien Husserl; la cible facile à identifier, même si l'auteur n'est pas expressément cité, est bien le "positivisme» antimétaphysique de Mach qui n'aura d'ailleurs jamais caractérisé en termes égologiques cet « originaire subjectif », puisque, aussi bien, une des thèses fondamentales et péremptoires de l'Analyse des sensations ${ }^{26}$ est que "das Ich ist unrettbar"-le Moi ne peut en aucune façon être sauvé. Il n'en est pas moins permis de s'interroger sur le point de savoir si et dans quelle mesure, de manière pour ainsi dire anticipée, cette critique natorpienne n'atteindrait pas aussi le Husserl des Ideen I quand celui-ci revendique pour le compte de la phénoménologie le terme même de positivisme:

Si par «positivisme» on entend l'effort, absolument libre de préjugé, pour fonder toutes les sciences sur ce qui est "positif », c'est-à-dire susceptible d'être saisi de façon originaire, c'est nous qui sommes les véritables positivistes.

[Positivisme» qui alors prend pour "point de départ] cela même qui est antérieur à tout point de vue, à savoir tout le champ du donné intuitif, antérieur même à toute pensée qui élabore théoriquement ce donné, tout ce qu'on peut voir et saisir immédiatement, à condition précisément qu'on ne se laisse pas aveugler par des préjugés et empêcher de prendre en considération des classes entières de données authentiques ${ }^{27}$.

Au-delà du compte rendu des Prolégomènes à la logique pure que Natorp publie sous le titre: Zur Frage der logischen Methode, dans les Kantstudien en 1901, c'est surtout dans l'Allgemeine Psychologie de 1912, et dans le compte rendu qu'il donne des Ideen en 1917-1918 ${ }^{28}$ que Natorp

26. Analyse der Empfindungen, Iéna 1903.

27. Ideen I, $\mathbb{S} 20$.

28. Allgemeine Psychologie nach kritischer Methode, Tübingen 1912; "Husserl's Ideen zu einer reinen Phänomenologie ", Logos, Internationale Zeitschrift für Philosophie der Kultur, VII, 1917/1918, pp. 224-246 (réédité in Husserl, H. Noack, éd., "Wege der Forschung ", Wissenschaftliche Buchgesellschaft, Darmstadt, 1973) 
revient sur la question du donné et de la double fondation, objective et subjective, de la connaissance.

Dans le chapitre XI de l'Allgemeine Psychologie, Natorp poursuit $(\mathbb{1 1})$ sa discussion des Recherches logiques pour souligner ce qui, à l'époque du premier compte rendu, avait été caractérisé comme "malaise logique », à savoir la tension qui subsiste entre le «formel » "pur » ou l' «idéal» et le réel, entendu comme "un résidu non-compris, irrationnel ("unbegriffener, unvernünftiger Rest»); "résidu étranger, réprouvé et pourtant inéliminable $»^{29}$. Pour sortir du malaise logique, il aurait fallu rétablir entre ces deux instances antagonistes: "l'existence supra-temporelle du logique» d'un côté, sa «factualité temporelle dans le vécu psychique» de l'autre, un lien logique, celui qui permettrait de donner tout son sens à l'idée d'une «Realisierung des Idealen », entendue comme «transition logique rigoureuse » d'un mode de considération à un autre, cette "transition " que Natorp nommera de son côté "objectivation", "reconstruction». En 1912 Natorp a alors pris connaissance de l'ensemble des Logische Untersuchung, ce qui n'était évidemment pas le cas à l'époque de son compte rendu des seuls Prolégomènes, et sa critique se fait alors plus aiguë: Husserl, qui semblait exiger, à juste titre, une «fondation strictement objective de la logique et de la connaissance objective en général » n'avait encore en réalité proposé qu'une fondation phénoménologique de la connaissance, c'est-à-dire une «fondation subjective et psychologique ». Mais, demande Natorp, même en s'en tenant à l'impératif de la description, comment celle-ci pourrait-elle échapper à l'objectivation propre à toute théorie? Si la réflexion sur le vécu psychique en fait nécessairement un objet, comment ce vécu, comment les actes intentionnels eux-mêmes (die meinende Akte) pourront-ils être appréhendés abstraction faite des expressions qui ne valent que pour les réalités qui sont visées ou intentionnées par eux? D'après Husserl, conclut Natorp, "la subjectivité est manifestement une deuxième objectivité de même nature que la première objectivité, celle à laquelle on pense habituellement, et qui lui est coordonnée.»

Or c'est là très précisément ce que Natorp entend refuser: la subjectivité, «envers» de l'objectivité, n'est pas «une deuxième objectivité », celle des «actes de conscience». Loin de toute description, la subjectivité, en tant qu'elle est l'envers ou l'image inversée (Gegenbild) de l'objectivité, ne saurait être atteinte que par une "voie indirecte", celle précisément qu'il faut nommer non pas Reduktion, mais bien Rekonstruktion. La démarche doit ici demeurer essentiellement indirecte, dans la mesure où l'objectivation réflexive des actes ou du subjectif en général ne peut être qu' «absolument non immédiate » et qu'elle demeure donc toujours «totalement dépendante de l'acte primaire de l'objectivation spécifique et originaire»(ibid., 281).

29. «[...] das Reale bleibt als fremder, verworfener, und doch nicht wegzuschaffender Rest stehen»(p. 14, reprint de la Wissenschaftliche Buchgesellschaft). 
Saisir le subjectif et les actes purement en eux-mêmes, "c'est ce que je n'ai pas réussi jusqu'ici", remarquait ironiquement Natorp à l'adresse de Husserl, même si Natorp accorde que cette opposition tranchée du subjectif et de l'objectif est susceptible de s'estomper dès lors qu'on est attentif au "caractère processuel» de l'"être". Quand on examine un vécu cognitif particulier, on s'aperçoit qu' «il n'y a pas en général du subjectif et de l'objectif en soi, mais que, dans la poursuite du procès de l'objectivation et donc aussi de la subjectivation, le caractère du subjectif et de l'objectif se transmet d'étape en étape à d'autres membres et encore à d'autres membres, le subjectif devient objectif et l'objectif redevient subjectif» (283).

Cette opposition du subjectif et de l'objectif, comme Natorp l'avait noté d'emblée, au seuil de l'Allgemeine Psychologie (p. 71), est en réalité tout à fait relative puisqu'elle nous renvoie finalement à un seul et même mouvement de pensée, susceptible d'être parcouru alternativement dans un sens, puis dans un autre:

Le rapport d'opposition (Gegensatz) devient un rapport de réciprocité (Gegenseitigkeit) qui signifie du même coup une corrélation (Korrelation) nécessaire. [...] Ce qui est ici décisif, c'est que le face-à-face du subjectif et de l'objectif, qui semblait au départ fixé, se dissout totalement dans le procès vivant de l'objectivation d'une part et de la subjectivation d'autre part, où n'existent ni objectif ni subjectif au sens absolu, mais toujours seulement des degrés relatifs d'objectif et de subjectif qu'on peut également caractériser avec un droit égal comme une différence de degré d'objectivation, ou, à l'inverse, de subjectivation ${ }^{30}$.

C'est naturellement la considération du caractère processuel de cet «être", terme que Natorp ne peut plus désormais mentionner qu'entre guillemets, qui est décisive: la connaissance doit être envisagée comme un processus par rapport auquel l'objet, toujours «à déterminer ", requiert une "fonction de connaissance », laquelle ne s'éclaire elle-même qu'après coup, sur la voie complémentaire de la subjectivation qui, elle aussi, se poursuit "dans l'indéterminé et à l'infini ». Voilà ce que Natorp présente au titre d'un examen «génétique» ou «dynamique» de la connaissance par opposition à tout examen "ontique» ou "statique». C'est cela qui définit le point de vue de la méthode par opposition à toute perspective axée sur un «résultat», fixe ou «figé ", ou encore la perspective du fieri, par opposition à toute prétention à aborder un factum ou un datum ultimes. Éviter de faire de l'objet un "en soi" ou un choriston, "se tenant debout tout seul à l'extérieur ", c'est aussi refuser de creuser le fossé sur un côté duquel se dresserait «le donné », "le subjectif », pensés de manière fixe et séparée (Allgemeine Psychologie, pp. 286-287). Ici, la critique de Husserl se fait plus nuancée. Comme le note en effet Natorp:

30. Cf. aussi, «Bruno Bauchs «Immanuel Kant» und die Fortbildung des Systems des Kritischen Idealismus », Kantstudien XXII, 1918, pp. 432-433. 
Husserl n'envisage pas la relation entre le contenu et l'objet, entre la présentation (Präsentation) et la représentation (Repräsentation) d'une manière fondamentalement différente de moi. Il reconnaît, du moins [...] à titre de cas idéal, que l'intention de signification et le «remplissement» sont absolument un, de sorte que l'objet même est renfermé dans le "contenu phénoménologique » (<LU. A, p. 549, 588-590>1). Nous dépassons cette position en soulignant le fait qu'un tel «remplissement» n'a pas lieu une fois pour toutes, mais à nouveau à chaque étape - qu'il n'y a jamais et qu'il ne peut pas y avoir non plus de remplissement absolu. Nous reconnaissons toutefois que Husserl s'approche peut-être au plus près de l'idéalisme lorsqu'il fait dépendre le contenu perceptif de la pensée, le «remplissement» de l'«intention", la présentation de la représentation, et qu'il détermine essentiellement le premier terme par le second - mais avec cette restriction: l'identification est "accomplie», mais elle n'est pas «visée» ( p. 622).

La perspective "génétique" conduit aussi à remettre radicalement en question l'idée même de vécu, et plus encore de "vécu originaire ", lequel, comme ne manque pas de le remarquer Natorp, est déjà caractérisé par Husserl lui-même comme ce qui est "aperçu dans la réflexion " ${ }^{32}$. Le prétendu "vécu originaire » devient ainsi à son tour un "problème »: non plus le thème d'une "description", mais bien d'une " reconstruction " ${ }^{33}$.

Dans cette nouvelle conception dynamique, précise-t-il encore:

il n'y a plus de donation figée du «contenu " et donc pas de possibilité d'une "description" directe de celui-ci-une telle description n'étant que le concept corrélatif d'une telle donation figée. Mais surgit à sa place la « reconstruction » qui n'est que l'envers de la construction de l'objet et qui partage avec elle le caractère génétique ou méthodologique, donc aussi le sens de l' «intention » et certes de l'intention qui ne se remplit jamais.

La partie critique de l'Allgemeine Psychologie concernant le privilège intacte, chez Husserl, de l'intuition et en particulier le motif de l'intuition eidétique se trouve reprise et approfondie dans le long compte rendu que Natorp donne, peu après, des Ideen I. En référence directe au fameux $\mathbb{S} 24$ qui formule le "principe de tous les principes ", Natorp insiste sur la filiation cartésienne de la phénoménologie husserlienne, ultimement indexée sur le donné directement offert à l'intuition. Dans sa réponse à la question du fondement de la certitude, qui ne saurait empiriquement renvoyer à l'expérience, Husserl — comme l'indique Natorp — "s'en tient à la position de Descartes » :

La connaissance de l'essence est fondée en tout premier lieu et principalement sur l'intuition. Celle-ci est caractérisée comme un voir immédiat, un intuitionner,

31. Hua. XIX, 2, 608, 645-648.

32. "Die Philosophie als strenge Wissenschaft», Logos, 1911 = Husserliana XXV, 29-30.

33. «La «description" devient nécessairement "reconstruction" ", Allgemeine Psychologie, p. 290. 
une «évidence» $[\mathbb{S} 3$, p. 12], une «vision de l'essence», une «saisie» au sein d'une immédiate évidence intellectuelle. [...] Tout ce que la phénoménologie établit apparaît comme prétendant être finalement donné directement dans l'«intuition » [ $\mathbb{S} 18$, p. 33], sans aucune adjonction d'hypothèse, sans aucun travail d'exégèse (ohne jede hypothetische oder interpretierende Auslegung oder Hindeutung) (réédition Darmstadt, p. 38).

Sans m’interroger ici plus avant sur le bien-fondé de cette critique, je signale simplement en passant que dans la réécriture qu'il propose du «principe des principes» (KNS, $\mathbb{S} 20$, pp. 109-110), cette caractérisation a bien pu jouer un rôle important pour le jeune Heidegger ${ }^{34}$. Quoi qu'il en soit, ce même Natorp ne manque évidemment pas de nuancer singulièrement sa critique en faisant remarquer que Husserl parle moins de donné ou d'êtredonné (Gegebensein), mais bien plutôt d' "acte originaire donateur", ou encore d' "intuition donatrice ", en excluant par là même l'idée qu'il puisse y avoir du donné au sens de la "simple réceptivité». Si le "donner» (das Geben) renvoie ainsi à un acte ou est un acte "tout à fait spécifique ", on peut alors se demander — et Natorp va évidemment jusque là — si «l'acte fondamental de la connaissance " n'est pas plutôt « un acte de poser» (Akt des Setzens), pour conclure, au terme d'une analyse qui traverse cette fois les déterminations platoniciennes et kantiennes, que (je cite un peu longuement le passage capital):

[...] l'acte de donner doit pouvoir — et peut effectivement ne rien signifier d'autre que: la fondation des positions singulières de la pensée, tout d'abord isolées, à partir de la continuité de la pensée et par elle.

Autrement dit, la fixité (Starrheit), le caractère ponctuel de la "vue » (Einsicht) prise dans son isolement, doivent être abolis et surmontés, mais en même temps expliqués par ce dépassement. La pensée est mouvement et non repos; les arrêts ne peuvent être que des processus, tout comme le point ne peut être "déterminé » que par le tracé de la ligne et non pas déterminé pour et par luimême avant ce tracé. [...]

34. «Das methodische Grundproblem der Phänomenologie, die Frage nach der Weise der wissenschaftlichen Erschließung der Erlebnissphäre, steht selbst unter dem «Prinzip der Prinzipien » der Phänomenologie. Husserl formuliert es so: «Alles, was sich in der "Intuition» originär... darbietet, [ist] einfach hinzunehmen ... als was es sich gibt.»Das ist das «Prinzip der Prinzipien ", an dem « uns keine erdenkliche Theorie irre machen» kann. Verstünde man unter Prinzip einen theoretischen Satz, dann wäre die Bezeichnung nicht kongruent. Aber schon, daß Husserl von einem Prinzip der Prinzipien spricht, also von etwas, das allen Prinzipien vorausliegt, woran keine Theorie irre machen kann, zeigt, daß es nicht theoretischer Natur ist, wenn auch Husserl darüber sich nicht ausspricht. Es ist die Urintention des wahrhaften Lebens überhaupt, die Urhaltung des Erlebens und Lebens als solchen, die absolute, mit dem Erleben selbst identische Lebenssympathie. Vorläufig, d.h. auf diesem Weg vom Theoretischen herkommend, in der Weise des immer mehr Sichfreimachens von ihm, sehen wir diese Grundhaltung immer, wir haben $z u$ ihr eine Orientirung. Dieselbe Grundhaltung ist erst absolut, wenn wir in ihr selbst leben - und das erreicht kein noch so weit gebautes Begriffssystem, sondern das phänomenologische Leben in seiner wachsenden Steigerung seiner selbst. » 
Si la pensée est mouvement, il faut questionner le factum sous l'angle du fieri et ne le reconnaître comme tel que dans la mesure où le fieri en est à son tour le fondement.

Telle est la raison pour laquelle nous n'acceptons aucune donation finie (fertige Gegebenheit), aucun tout-fait <en français dans le texte>, qu'il soit a priori ou empirique. Les prétendues «étoiles fixes» de la pensée doivent être reconnues comme des «astres errants d'un ordre plus haut », les prétendus points fixes de la pensée doivent se résoudre, se liquéfier dans la continuité du procès de la pensée. Ainsi rien n'est "donné», mais quelque chose seulement devient «donné» (So ist nichts, sondern wird etwas "gegeben») (CR. Ideen I, éd. cit., 42).

Ainsi, c'est l'idée même de donation ou de donné ultime qu'il faut récuser absolument: même une "position » ne saurait être considérée comme ultimement et absolument donnée. De donation il n'y a que dans le "procès » du penser et par lui, et il est donc légitime d'en conclure que «Der Prozeß selbst ist das "Gebende », qu'il n'y a pas d'instance donatrice à proprement parler, et que, ce qui donne, c'est le procès ou la processualité. Dans l'article qu'il consacre en 1918 au Kant de Bruno Bauch, Natorp mettra également en lumière ce caractère actif $\mathrm{du}$ "donner » à l'arrière-plan de toute donation: «Es muß der Gegebenheit ein aktives Geben entsprechen ${ }^{35}$. À tout être-donné, à toute donation doit nécessairement correspondre un "donner actif».

«Nurso «gibt» es, "gibt sich» [...] Gegebenes». C'est seulement ainsi qu'il y a ou que se donne du donné. Le jeu de mots aura ici aussi certainement retenu l'attention de Heidegger qui demandera quelques mois plus tard, dans son premier cours de l'année 1919, en radicalisant la question: "gibt es etwas? ", y a-t-il quelque chose <de donné> ? pour la reformuler en ces termes "Gibt es das 'es gibt'? ", y a-t-il le «il y a " ? comme est donné le «ça donne»? (Ga. 56/57; 62-63).

$\mathrm{Si}$, selon la démarche réductive de la phénoménologie husserlienne dans les Ideen, la conscience pure, ou mieux la "région conscience », devient la dernière "sphère de position absolue » ( $\mathbb{4} 46)$, celle qui est séparée par "un abîme de sens" de la "réalité ", cette région ontologique où l'être s'esquisse, sans jamais être donné absolument $(\$ 49)$, par opposition à la régionconscience qui demeure, au terme de la réduction phénoménologique, comme l'ultime "résidu phénoménologique» donné de façon absolu, il importe, aux yeux de Natorp, de substituer à cette réduction, qui n'est en vérité qu'une "simple omission de l'acte de position objective" (das bloße Unterlassen des gegenständlich setzenden Aktes), la «reconstruction»: celle-ci, à l'inverse de toute tentative visant à accéder à la conscience dégagée dans sa "pureté ", comme "système d'être fermé sur soi ", "système d'être absolu dans lequel rien ne peut pénétrer et duquel rien ne peut s'échapper» (\$49), se présente comme une autre tâche spécifique, exigeant une «méthode 
appropriée ", directement opposée à l'orientation naturelle de la connaissance objective ${ }^{36}$. Natorp peut bien accorder à Husserl que "cette méthode est exactement inverse de la méthode d'objectivation", mais - et c'est par là que la séparation d'avec la démarche phénoménologique est sans retour -, cette méthode doit demeurer «dans la correspondance la plus exacte avec elle <l'objectivation> ", par où, ajoute Natorp, "elle ouvre une voie au sein de l'infini » (op. cit., p. 49). C'est par là et par là seulement qu'il devient possible de «reconduire toutes les objectivations" à une "conscience originaire ", laquelle (nous l'avons vu) ne saurait jamais être absolument donnée, ni constituer à proprement parler une "source", puisque aussi bien son " originarité » prétendue (Ursprünglichkeit) se confond ici avec son «caractère d'être-fondée-en-soi-même» (in sich-selbst-Gegründetheit), ce caractère qui n'appartient en propre qu'au seul "penser ", étant entendu que l'être-ensoi-même ici «signifie et ne peut signifier que: im Prozeß sein, être dans le procès ». Nous retrouvons ici, avec un sens plus riche, la formule déjà citée: "l'absolument et ultimement donné, ne peut être «donné » que dans et par le procès du penser ": Der Prozeß selbst ist das "Gebende» — le procès luimême est l'instance "donatrice».

En mettant ainsi l'accent sur la processualité du penser, en rappelant la différence radicale que Husserl aurait méconnue entre présentation (Darstellung) de la conscience pure d'un côté, et mise en lumière de «ce qui peut être présenté (dargestellt) dans une connaissance effective » de l'autre, Natorp peut même se payer le luxe d'insister, contre l' «abstraction » de la démarche husserlienne qui est en réalité un "refus de prendre en considération l'ensemble du monde de l'objectivité", sur l'entrelacement ou l'intrication infinie Verflechtung) de la conscience et de ses actes objectivants, en se faisant ainsi le défenseur du «flux» que Husserl se verrait contraint d' "arrêter» ou de "fixer ", sans parvenir à rester fidèle à la thèse pourtant reconnue que "originairement, c'est le temps qui est dans la conscience et non pas la conscience qui est dans le temps» (Allgemeine Psychologie, 228).

Avant d'abandonner ici Natorp, je voudrais encore - et ce n'est pas simplement un point de curiosité - évoquer la position du dernier Natorp relativement à la question du "donné », du «il y a » (es gibt), du «factum », qui n'est plus à présent le factum de la science constituée, à partir duquel il importait de revenir, par «reconstruction», jusqu'au foyer inassignable de

36. Cf. Allgemeine Psychologie, op. cit., p. 194. En ce sens, et comme Natorp le notait dès l'Einleitung in die Psychologie, p. 94, la reconstruction est indexée sur la réflexion: "Que la reconstruction représente une tâche réelle et pas du tout facile, c'est-ce qui ressort clairement si l'on considère comment, immédiatement et de manière inaperçue, s'accomplit d'ordinaire l'objectivation. Déjà toute dénomination, toute concentration du regard [...], bref toute fonction orientée, fût-ce de loin, sur la connaissance implique un essai d'objectivation. Sans une réflexion tout à fait spécifiquement orientée sur la subjectivité de l'apparaître (auf die Subjektivität des Erscheinens gerichtete Reflexion), nous ne prêtons attention qu'à l'objet, nous objectivons." 
la conscience objectivante et constituante. Dans les Leçons qu'il donne à Marburg en 1922-1923, et qui ne seront publiées à titre posthume qu'en 1958, sous le titre Philosophische Systematik ${ }^{37}$, une section tout entière est consacrée aux "catégories de l'individuation» (Section C, $\$$ 62, pp. 223 sq.).

Natorp prend encore pour point de départ un Faktum singulier, mais c'est cette fois le factum qu'il $y$ a, ou mieux le factum du «es gibt »: «es gibt das Faktum, es gibt: das 'es gibt'». Qu'en est-il de la factualité (Faktizität) de ce fait singulier qui se confond à présent avec une ultime et irréductible «donation »: « il y a ", cet «il y a » qui tout à la fois donne et est donné ?

Le «il y a ", c'est ici aussi bien l'ultime "c'est»: "es ist, das es ist». Qu'en est-il de ce Sein qui semble désormais prendre le pas sur tout procès, sur tout fieri? Natorp en souligne l'unicité (Einzigkeit) irréductible: unicité qu'il n'y a plus lieu de produire ou d'attester (anfweisen) à travers quelque développement (Entwicklung) si poussé soit-il; unicité qui ne se laisse pas davantage fonder en raison ou sonder (begründen, ergründen), car c'est elle en effet qui constitue l'ultime sens de l'être (Sinn des Seins), le dernier sens à mettre en valeur et auquel on puisse accéder et mettre en valeur. Ultime unicité que Natorp caractérise aussi, de manière assez énigmatique, comme celle de la Sache, la "Chose », ou l'affaire: «...die Sache...die letzte Einzigkeit» (op. cit., 227). Cette "Chose» unique qui est, nous dit Natorp, la "merveille la plus étonnante", celle qui régit de part en part «le tout de l'être et du sens"; merveille qui tient aussi à ceci que, ici, "ce qui est le plus lointain» est également, en un sens, "le plus proche", celui qui ne saurait jamais être rejoint à travers quelque médiation que ce soit, même la plus sublime, et qui est plutôt comme "das unmittelbar Vorliegende», ce qui est là présent-devant immédiatement; cela qui «mettant toutes choses en question» (dies alles unter Frage Stellende) demeure lui-même définitivement in-questionnable, hors-de-question (das schlechthin Unfragliche). Sache, singulière et unique encore une fois, qui est destinée à demeurer le véritable point de départ, notre seule issue (Ausgang) incontournable: das schlichte Daß, das schlichte «es ist»: das Faktum (ibid., 227) — «le pur et simple quod, le pur et simple "c'est», le factum».

Rien, strictement rien ne nous permet de penser que le jeune Heidegger qui commence à Fribourg son enseignement auprès de Husserl en 1919, ait pu connaître d'une façon ou d'une autre, la teneur des réflexions du vieux Natorp, réflexions dont il faudrait par ailleurs interroger le degré d'inconséquence ou d'incohérence par rapport aux critiques formulées une vingtaine d'années auparavant à l'encontre d'une forme très définie du «mythe du donné ». Quoi qu'il en soit de ce dernier point, dans l'examen duquel je ne me lancerai pas, il n'est pas du tout nécessaire de formuler une hypothèse de ce genre, très invraisemblable d'ailleurs, pour comprendre comment le jeune Heidegger, dans un geste de réappropriation critique de la phénoménologie

37. Philosophische Bibliothek, Meiner Verlag, Hambourg. 
husserlienne, a pu relancer à son tour selon des formulations et dans un horizon tout à fait renouvelés la question du donné ou mieux du «es gibt ».

Au plan des "sources", si quelque chose de tel existe et importe ici, il suffirait en effet de renvoyer à Emil Lask et en particulier à son ouvrage de 1911, Die Logik der Philosophie und die Kategorienlehre, si massivement présent dans l'écrit d'Habilitation consacré à Duns Scot, sa doctrine des catégories et de la signification. Emil Lask en effet consacre dans cet ouvrage plusieurs longues analyses au « es gibt » au titre des «catégories de la réflexion ». Mais c'est là un autre chapitre que je ne vais pas aborder aujourd'hui, me bornant à risquer une dernière hypothèse, celle selon laquelle le propos heideggérien de la Destruktion, "destruction » ou "déconstruction ", s'il hérite bien en un sens de l'Abbau husserlien, de la désédimentation de strates accumulées qui sont venues obstruer la saisie intuitive (l'Einsicht), ce propos pourrait tout aussi bien se comprendre comme la reprise, à un renversement près, de la Rekonstruktion, de la reconstruction natorpienne. Quant au projet général de la «reconstruction", Heidegger note bien qu'il «s'agit avec la reconstruction d'un renversement total et complet du procédé de la connaissance objectivante ", procédé qui vise à accéder " au flux du vécu », au "sujet ultime de la conscience», au "vécu immédiat», au "contexte concret du vécu originaire». Par là même, la «reconstruction" semble bien présenter plus que des affinités avec ce que Heidegger nomme d'emblée la "méthode » de la «destruction" pour autant que celle-ci vise à «reconduire la philosophie à elle-même à partir de son extériorisation ». La «déconstruction » $(A b b a u)$ vise en effet à "se libérer d'une tradition inauthentique qui s'impose à nous de manière non originaire (nicht ursprünglich zugeeignet » (GA. $59,5)$, et, bien loin d'être une destruction purement négative (Zerschlagen und Zertrümmern), elle est «dijudication» (Diiudication) (GA. 59, 74):

la destruction consiste fondamentalement en un acte de «discernement» : elle a pour première fonction d'opérer le « discernement entre ce qui, du point de vue phénoménologique, doit être considéré comme originaire et non originaire (ursprünglich - nichtursprünglich).

Mais quand on en vient à la question décisive (GA. 56/57, 108) que Heidegger formule en ces termes: «Nous posons la question: la méthode de la reconstruction effectue-t-elle ce qu'elle est censée effectuer? Peut-elle, en général, l'effectuer? ", la réponse est clairement: "Non", dès lors qu'avec la reconstruction on n'a jamais quitté le terrain de l'objectivation: "La reconstruction est, elle aussi, construction, et être constructif, tel est justement ce qui caractérise en propre l'objectivation - qui est comme telle théorétique» (ibid. $)^{38}$. C'est pourquoi, comme le note encore Heidegger (GA. 59,

38. Cf. aussi GA., 59, 105: «Die Psychologie kann nichts rekonstruieren, was nicht zuvor konstruiert ist. Inhaltlich und umfänglich decken sich bezüglich des zu Erforschenden Objektivierung und Subjektivierung, nur die Richtung ist diametral entgegengesetz. Das Logische (Objektive) bleibt immer die Gegenseite alles Psychischen (Subjektiven).» 
96): «La considération destructive, caractéristique de la position de Natorp, est nécessaire et féconde pour plusieurs raisons. [...] Cette position recherche, selon son propre sens, "l'origine" avec une intensité et une radicalité à la mesure de son fourvoiement. » C'est, on l'aura compris, un compliment! Le problème qui doit donc être repris ou répété là où Natorp l'a laissé, c'est précisément celui de la description, dont l'ultime possibilité est toujours chez Husserl assignée à l'intuition, conformément au "pacte " phénoménologique: dire, décrire ce qui est vu. S'il est permis d'affirmer qu' "un pas décisif a été accompli par la phénoménologie: l'accent mis sur l'intuition originaire (originäre Anschauung) — évidence! — et l'idée de la description adéquate ", il convient pourtant d'opposer au privilège accordé par Husserl à l'intuition l'idée d'une coappartenance indissociable de l'intuition et de la compréhension, l'idée d'une verstehende Anschauung. C'est dans cette perspective là que s'impose résolument la démarche de la Destruktion, car en effet ce qui est caractéristique de la "vie facticielle », c'est précisément "l'effacement de la significativité » (das Verblassen der Bedeutsamkeit) (GA. 59, 37) qui pèse sur elle comme une menace permanente - effacement de la significativité, déperdition de la signification concrète et contextuelle qui tient au fait qu'un sens n'est plus «accompli», qu'il est donc amputé de la dimension intentionnelle spécifique que lui confère à chaque fois le Vollzugssinn. C'est à ce Vollzug, prestation ou accomplissement effectif qu'entend revenir la déconstruction. Par là, elle entreprend, elle aussi, un retour en amont, une remontée jusqu'à la source donnante; mais celle-ci demeure par principe inatteignable, ou plutôt ne saurait être qu'approchée à l'horizon d'une démarche qui traverse moins les "objectivations " que les sédiments, les couches de sens fixés ou figés théorétiquement, effacés et dissimulant ainsi non plus un procès, mais un Vollzug, une effectuation à chaque fois concrète et facticielle, qui constitue le véritable foyer de toute signifiance: das Bedeutsame, die Bedeutsamkeit. 